\title{
Reflets
}

Revue ontaroise d'intervention sociale et communautaire

\section{Des nouvelles du comité francophone provincial de l'Association provinciale de travailleurs sociaux}

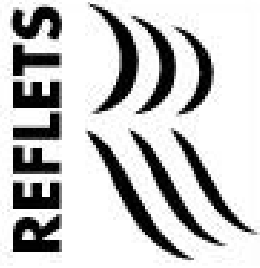

\section{Lise Andrée Payette}

Volume 1, numéro 1, printemps 1995

Des pratiques à notre image : défis et ressources

URI : https://id.erudit.org/iderudit/026070ar

DOI : https://doi.org/10.7202/026070ar

Aller au sommaire du numéro

Éditeur(s)

Reflets : Revue ontaroise d'intervention sociale et communautaire

ISSN

1203-4576 (imprimé)

1712-8498 (numérique)

Découvrir la revue

Citer cet article

Payette, L. A. (1995). Des nouvelles du comité francophone provincial de l'Association provinciale de travailleurs sociaux. Reflets, 1(1), 233-237.

https://doi.org/10.7202/026070ar

Tous droits réservés (C) Reflets : Revue ontaroise d'intervention sociale et communautaire, 1995
Ce document est protégé par la loi sur le droit d'auteur. L'utilisation des services d'Érudit (y compris la reproduction) est assujettie à sa politique d'utilisation que vous pouvez consulter en ligne.

https://apropos.erudit.org/fr/usagers/politique-dutilisation/ 


\title{
Des nouvelles du comité francophone provincial de l'Association provinciale de travailleurs sociaux
}

\author{
Lise Andrée Payette \\ Coordonnatrice du Comité francophone provincial de l'ATSPO
}

\section{Un peu d'histoire}

Le Comité francophone provincial est établi par l'Association des travailleurs sociaux professionnels de l'Ontario (ATSPO) en 1983 pour répondre aux besoins de ses membres. En mai 1986, l'ATSPO se déclare officiellement bilingue et adopte une politique globale de bilinguisme. «C'est le Comité francophone provincial qui a rédigé cette politique, approuvée par le conseil d'administration en octobre 1987. Cette politique énonce les principes de communiquer, par écrit et oralement au niveau interne et au niveau externe.» [Lalonde, 1991:37]. L'adoption d'une politique de bilinguisme à l'ATSPO a été influencée par l'entrée en vigueur imminente de la Loi 8, qui reconnait de façon officielle certains droits aux francophones de l'Ontario.

À la réunion annuelle du 22 mai 1992, les membres du Comité francophone provincial approuvent une nouvelle structure organisationnelle visant à favoriser une plus grande participation des membres par l'entremise de groupes de travail. Les objectifs 
Reflets

principaux sont de développer une meilleure communication entre les membres francophones au sein de l'ATSPO et d'encourager une participation axée sur leurs besoins et leurs intérêts professionnels.

\section{Tâches du comité francophone provincial}

Afin d'assurer un fonctionnement efficace, le Comité assume les tâches permanentes suivantes:

- préparer les demandes de subvention pour chaque année fiscale

- s'assurer d'une participation active des francophones au sein des comités provinciaux de l'Association

- évaluer les politiques de bilinguisme au sein de l'Association et assurer le suivi de dossiers qui en découlent lorsqu'il y a lieu

- organiser la réunion annuelle du Comité provincial francophone

- assurer une représentation francophone au sein du Comité de direction et du Conseil d'administration de l'Association

- établir et maintenir une banque de ressources francophones

- préparer un budget annuel et fournir régulièrement des états financiers

- encourager la formation de groupes de travail spontanés, selon les besoins et les intérêts professionnels des membres

- soutenir les activités des groupes de travail.

Les fonds de fonctionnement du Comité proviennent de l'ATSPO et du ministère des Services sociaux et communautaires. De plus, depuis septembre 1992, une infusion de fonds provenant du Regroupement des intervenantes et intervenants francophones en santé et en services sociaux de l'Ontario (RIFssSO) permet un plus grand déploiement d'initiatives à l'échelle provinciale, notamment au niveau des groupes de travail. 


\section{Groupes de travail}

Des critères pour déterminer la nature et les modalités de ces groupes de travail sont établis et le Comité est à mettre au point un document à cet effet qui sera disponible à la réunion annuelle de novembre 1995. Il est à noter toutefois que les groupes de travail qui reçoivent des fonds doivent soumettre un article pour publication dans la revue provinciale Le Bulletin. Ceci renseigne les membres au sujet des activités qui se déroulent en français et encourage par le fait même, la rédaction d'articles en français.

Durant l'année fiscale 1992-1993, des projets dans le cadre de ces groupes de travail sont mis sur pied à Timmins, à Sudbury et à Ottawa.Voici une brève description de ces initiatives:

\section{Santé et mieux-être}

Les 22 et 23 octobre 1992 et le 15 janvier 1993, Madame Denyse Dupont-Ryan offre un atelier de trois (3) jours intitulé Santé et mieux-être aux intervenantes et intervenants francophones de la région de Timmins. Cette activité s'avère un succès, injectant aux participantes et participants une énergie renouvelée.

\section{Revue ontaroise}

Les fonds accordés dans le contexte de ces groupes de travail ont facilité le démarrage de la Revue ontaroise d'intervention sociale et communautaire. L'objectif de ce groupe de travail, composé de professeures et de professeurs des écoles de service social de l'Université Laurentienne et de l'Université d'Ottawa, était d'évaluer, par le biais d'un sondage, l'intérêt suscité par une revue s'adressant aux intervenantes et intervenants francophones et la pertinence de poursuivre un tel projet. Ce sondage a donné les résultats suivants: $97 \%$ des répondantes et répondants ont affirmé qu'une telle revue serait utile; $90 \%$ sont prêts à s'y abonner; $48 \%$ se proposent de soumettre des articles et $21 \%$ sont prêts à participer au comité de rédaction. 
Reflets

L'intervention de groupe auprès des populations vulnérables: défis actuels

Le 27 mars 1993, une quarantaine d'intervenantes et d'intervenants francophones participent à un colloque sur l'intervention de groupe, dans le cadre duquel les participantes et participants soulignent l'importance de créer des liens, briser l'isolement et promouvoir la solidarité. De cette rencontre émerge la suggestion de faire de ce colloque un événement annuel. Au cours de l'année fiscale 1993-1994, les projets des groupes de travail s'organisent dans les mêmes régions que l'année précédente. Le regroupement des intervenants francophones de Timmins offre, le 25 mars 1994, une journée d'ateliers animés par des personnes-ressources du milieu, autour des trois thèmes suivants: les lois et l'intervenant, la programmation neurolinguistique (P.N.L.) et les perspectives holistiques sur la toxicomanie, reliées à l'intervention. Les commentaires d'évaluation ont démontré que la journée avait été un véritable succès. On souligne aussi que tous les membres de l'ATSPO bénéficient d'un tel projet qui fait aussi connaitre l'Association et les avantages qu'elle offre aux non-membres. (Lamontagne, 1994)

\section{Encourager l'écriture}

Ce projet proposé par le groupe de Sudbury fait suite à la demande initiale portant sur la création d'une revue ontaroise et propose la réalisation d'un guide de rédaction à l'intention des membres francophones de l'ATSPO pour démystifier l'écriture et encourager les gens à participer à la revue.

\section{Intervenir en français chez nous en Ontario}

Plus d'une soixantaine de personnes d'Ottawa et de l'est ontarien participent à ce colloque tenu en avril 1994. Le comité organisateur s'était fixé deux objectifs principaux, soit de susciter un mécanisme d'échange facilitant l'analyse du contexte d'intervention auprès de la population francophone d'OttawaCarleton et de confronter les données de la rechercheintervention portant sur différentes problématiques spécifiques 
au milieu francophone ontarien aux réalités de la pratique auprès de cette population. Ce second objectif était lié aux projets de recherche-intervention des étudiantes et étudiants de la deuxième année du programme de maitrise en service social de l'Université d'Ottawa, contribuant ainsi à tisser des liens entre l'École et la communauté (Dubois, 1994).

Enfin, pour l'année en cours, des projets s'organisent dans les chapitres du nord-est, du sud-centre/sud-ouest et d'Ottawa.

\section{Les membres du comité francophone provincial}

Le comité francophone provincial se compose présentement des cinq personnes suivantes: Roger Marrec de Toronto, Lise A. Payette d'Ottawa, Lyne Poliquin de Hearst, Gilles Renaud d'Alexandria et notre dernier membre, Marc Rouillé de la région de Welland. Ces personnes se rencontrent au moins deux fois par année et tiennent deux à trois téléconférences pour mettre à jour les activités du Comité.

Grâce au dévouement des membres fondateurs et anciens membres, le comité actuel continue, par son engagement et sa disponibilité, à travailler pour les membres francophones de l'ATSPO. Il compte sur l'appui du bureau de Toronto et en particulier de Rita Mascherin, adjointe de direction, pour assurer une liaison et une continuité des activités francophones au sein de l'Association des travailleurs sociaux professionnels de l'Ontario.

\section{Bibliographie}

DUBOIS, Madeleine (1994). «L'intervention en français chez-nous en Ontario: réflexions d'un colloque», Bulletin, vol. 21, no 4, Toronto.

LALONDE, Jocelyne (1991). Les enjeux de la mise en oeuvre du bilinguisme au sein d'une association professionnelle en Ontario, Mémoire de maitrise, École de service social, Université Carleton, Ottawa.

LAMONTAGNE, Claudette (1994). «Un colloque réussi! Des thèmes variés pour les intervenants(es) francophones», Bulletin, vol. 21, no 3, Toronto. 